\title{
Embryonic Primary Head Sinus may Persist in the Petrosal Bone
}

Original

Article

Yutaka Mitsuhashi, ${ }^{1}$ Koji Hayasaki, ${ }^{2}$ Taichiro Kawakami, ${ }^{3}$ Takashi Nagata, ${ }^{1}$ Yuta Kaneshiro, ${ }^{4}$ Ryoko Umaba, ${ }^{3}$

Toshiyuki Sugino, ${ }^{1}$ and Tsuyoshi Inoue ${ }^{1}$

Objective: To report on unusual veins traversing the petromastoid part of the temporal bone (petrosal bone) and to discuss their embryological origins.

Methods: Unusual veins traversing the petrosal bone were incidentally found on CTA, MRI, or conventional angiography in four cases. We have evaluated the course of these veins in detail and have reviewed the previous descriptions in the literatures about similar venous variations as well as the osseous and venous embryology around the petrosal bone.

Results: In all cases, the vein was anteriorly connected to the dural venous sinus around the foramen ovale and entered the petrosal bone through the facial hiatus. With regard to the subsequent running course and its exit from the petrosal bone, the vein crossed the petrous internal carotid artery, exited the petrosal bone into the petroclival fissure, and entered the inferior petrosal sinus in two cases. In one case, the vein exited the petrosal bone through the stylomastoid foramen after running the entire length of the facial canal. In the remaining case, the vein ascended in the petrosal bone along its anterior aspect and emptied into the superior petrosal sinus. The running course of these veins may correspond to the course of the embryonic primary head sinus and its tributaries.

Conclusion: Here we report on rare venous channels in the petrosal bone. We also believe that these veins may be remnants of the embryonic primary head sinus, based on their course in the petrosal bone and the embryological development of the veins in the region.

Keywords > aberrant vein, embryology, petrosal bone, primary head sinus

\section{Introduction}

In the early embryonic stages, the venous drainage of the head region is carried by a pair of venous channels, called the primary head sinus by Padget ${ }^{1}$; the primary head sinus

${ }^{1}$ Department of Neurosurgery, Ishikiri-seiki Hospital, Higashiosaka, Osaka, Japan

${ }^{2}$ Department of Neurosurgery, Japan Community Health Care Organization, Hoshigaoka Medical Center, Hirakata, Osaka, Japan

${ }^{3}$ Department of Neurosurgery, Osaka City University Graduate School of Medicine, Osaka, Osaka, Japan

${ }^{4}$ Department of Neurosurgery, Shimada Municipal Hospital, Shimada, Shizuoka, Japan

Received: May 20, 2016; Accepted: August 3, 2016

Corresponding author: Yutaka Mitsuhashi. Department of Neurosurgery, Ishikiri-seiki Hospital, 18-28 Yayoi-cho, Higashiosaka, Osaka 579-8026, Japan

Email: y-mitsuhashi@mtf.biglobe.ne.jp

(C)2016 The Editorial Committee of Journal of Neuroendovascular

Therapy. All rights reserved. is situated ventrolaterally to the primitive brain and continuous to the anterior cardinal vein (future internal jugular vein). Along with the development of the dorsal secondary venous pathways (the superior sagittal, transverse, and sigmoid sinuses), the original ventral venous drainage through the primary head sinus attenuates, particularly in the area ventral to the otic capsule [future petromastoid part of the temporal bone (petrosal bone)]. The fate of the regressed embryonic primary head sinus has been a matter of great interest. Incidentally we found unusual veins in the petrosal bone, which may be the remnants of the embryonic primary head sinus. However, there is little literature on similar venous variations in the petrous bone. Here we discuss the validity of the hypothesis that those veins are remnants of the primary head sinus.

\section{Materials and Methods}

Between January 2009 and February 2015, we found an unusual vein traversing the petrosal bone on CTA, MRI, or 


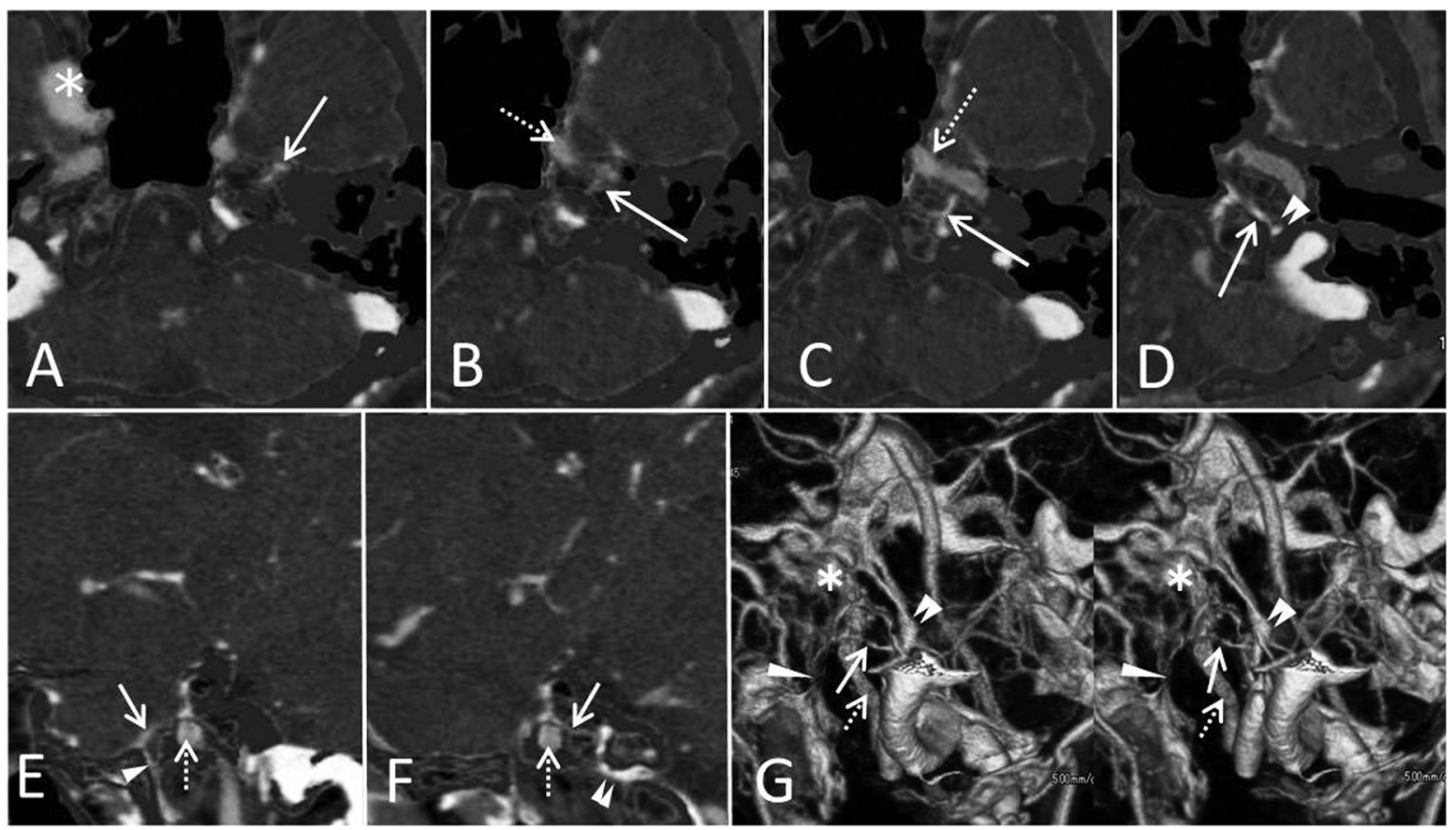

Fig. 1 Case 1. Axial bone-subtracted CTA around the left petrosal bone, from cranial (A) to caudal (D). (A) An unusual vein (arrow) posteriorly runs from the vicinity of the foramen ovale to enter the petrosal bone through the facial hiatus. Asterisk: right cavernous carotid aneurysm. (B, C) The vein (arrow) medially crosses over the petrous internal carotid artery [ICA (dotted arrow)] and exits the petrous apex into the petroclival fissure. (D) The vein (arrow) runs through the petroclival fissure to empty into the caudal part of the inferior petrosal sinus [IPS (double arrowhead)]. Oblique sagittal images reconstructed parallel to the course of the unusual vein in the left petrosal bone, from lateral $(\mathbf{E})$ to medial $(\mathbf{F})$. (E) The vein (arrow) is anteriorly connected to the emissary vein of the foramen ovale (arrowhead). Dotted arrow: petrous ICA. (F) The vein (arrow) posteromedially runs in the petrous apex crossing over the petrous ICA (dotted arrow) to flow into the IPS (double arrowhead). (G) Three-dimensional reconstructed images [left oblique posterosuperior view (stereogram)]. The vein (arrow) lies between the vicinity of the emissary vein of the foramen ovale (arrowhead) and the IPS (double arrowhead) across the ICA (dotted arrow). Asterisk: cavernous sinus; ICA: internal carotid artery; IPS: inferior petrosal sinus

conventional angiography in four patients (one men and three women; age range: $35-78$ years, mean age: 62.3 years) during evaluations for other intracranial disorders (two cerebral aneurysms, one glioma, and one cavernous dural arteriovenous fistula). We evaluated the course of those veins in detail and the relationship with the adjacent venous structures and reviewed previous literature that had described these venous variations or the osseous and venous embryology of the petrosal bone.

\section{Results}

In all four patients, the vein was anteriorly connected to the dural venous sinus around the foramen ovale and entered the petrosal bone through the facial hiatus. With regard to the subsequent course and its exit from the petrosal bone, the vein ran through the petrous apex, exited the petrosal bone into the petroclival fissure, and entered the inferior petrosal sinus (IPS) in two cases. In one case, the vein exited the petrosal bone through the stylomastoid foramen after running the full length of the facial canal. In the remaining case, the vein ascended in the petrosal bone to empty into the superior petrosal sinus (SPS). In all cases, there were no symptoms related to those veins or other congenital anomalies of the ear or venous structures in or around the petrosal bone.

\section{Cases}

\section{Case 1}

A 75-year-old female presented with diplopia due to right abducens palsy. A bone-subtracted CTA (Fig. 1) confirmed a carotid aneurysm in the right cavernous sinus. The aneurysm appeared to be responsible for the abducens nerve palsy. Although we did not think that it contributed to the patient's symptoms, we also found an unusual vein that penetrated the left petrosal bone. It entered the petrosal bone through the facial hiatus and crossed over the internal carotid artery (ICA) in the petrous apex and exited the petrous bone into the petroclival fissure to empty into the caudal part of the IPS. 


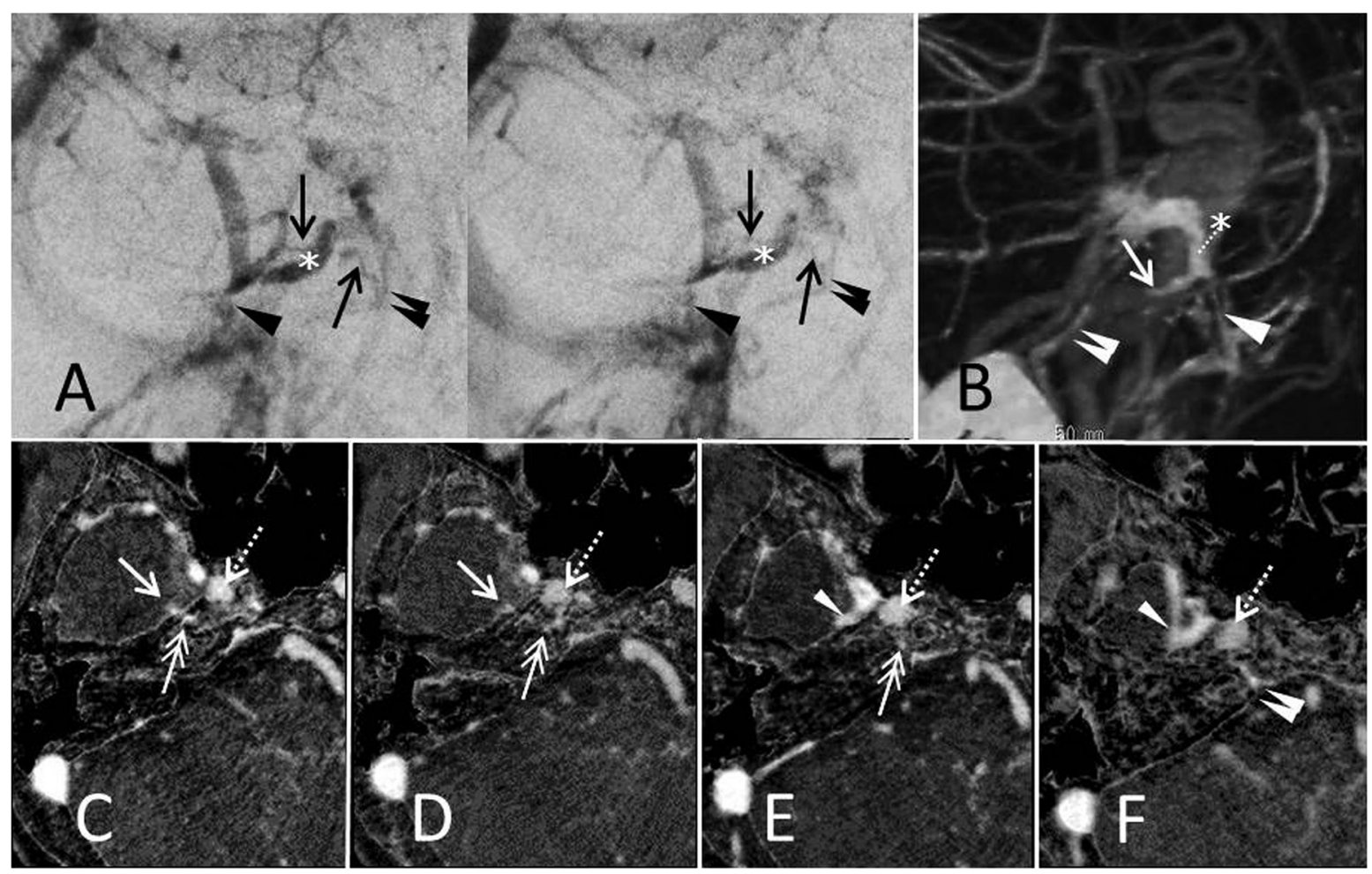

Fig. 2 Case 2. (A) Venous phase of the right carotid angiogram [anterior-posterior view (stereogram)] shows a vein (arrows) connected to the lateral part of the cavernous sinus (asterisk) near the emissary vein of the foramen ovale (arrow head) to the caudal part of the inferior petrosal sinus [IPS (double arrowhead)]. (B) Lateral view of maximum intensity projection of bone-subtracted CTA shows that the vein (arrow) anteriorly connected to the lateral part of the cavernous sinus (asterisk) near the emissary vein of the foramen ovale (arrowhead) posteriorly runs toward the caudal part of the IPS (double arrowhead). Axial bone-subtracted CTA around the right petrosal bone, from cranial $(\mathbf{C})$ to caudal $(\mathbf{F})$, shows that the vein anteriorly connected to the lateral part of the cavernous sinus (arrowhead) near the foramen ovale enters the petrosal bone through the facial hiatus (arrow), traverses the petrous apex (double arrow) across the internal carotid artery (dotted arrow), and flows into the IPS (double arrowhead). IPS: inferior petrosal sinus

\section{Case 2}

A 78-year-old female presented with conjunctival chemosis of the left eye and diplopia due to left abducens palsy; we suspected that she had a carotid cavernous fistula. The cerebral angiography revealed a left cavernous dural arteriovenous fistula that may have been responsible for the patient's symptoms. On the venous phase (Fig. 2A) of the right carotid angiogram, we found an unusual vein that ran from the lateral part of the cavernous sinus near the foramen ovale to the IPS. The bone-subtracted CTA (Figs. 2B-2F) showed that the vein ran through the facial hiatus and crossed over the petrous ICA to exit the petrosal bone into the petroclival fissure, and emptied into the caudal part of the IPS. The dural arteriovenous fistula was localized on the left side, and there was no shunting drainage to the right side; therefore, the dural arteriovenous fistula did not appear to be related to the unusual vein in the right petrosal bone.

\section{Case 3}

A 35-year-old male presented with a partial seizure of the right side of the face. MRI revealed a glioma in the left frontal lobe. An unusual enhancement through the entire length of the right facial canal from the facial hiatus to the stylomastoid foramen was found on contrast-enhanced T1-weighted MRI (Figs. 3A-3E). The venous phase of the right carotid angiogram (Figs. $\mathbf{3 F}$ and $\mathbf{3 G}$ ) showed that a venous channel from the middle fossa dural sinus near the emissary vein of the foramen ovale ran in the petrosal bone, descended the lateral side of the internal jugular vein, and emptied into the external jugular vein. This venous channel appeared to correspond to the unusual enhancement through the facial canal on MRI.

\section{Case 4}

A 61-year-old female was referred for endovascular embolization of an unruptured anterior communicating artery 

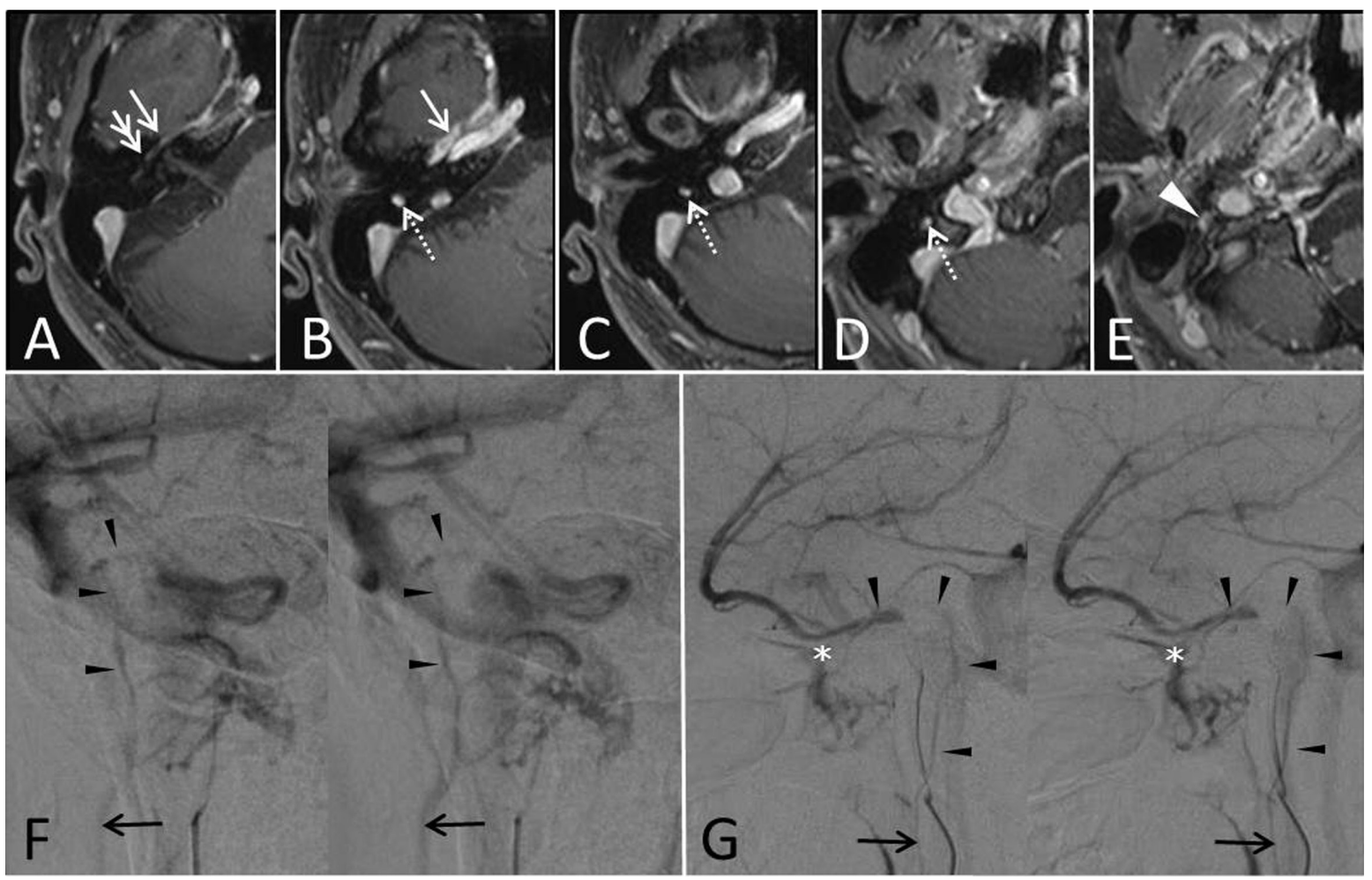

Fig. 3 Case 3. Axial contrast enhanced T1-weighted MRI around the right petrosal bone, from cranial (A) to caudal (E), shows an unusual enhancement through the full length of the facial canal, from the facial hiatus (arrow) to the stylomastoid foramen (arrowhead). Double arrow: tympanic segment of the facial canal. Dotted arrow: vertical segment of the facial canal. Venous phase of right carotid angiogram $[(\mathbf{F})$ anterior-posterior view (stereogram), $(\mathbf{G})$ lateral view, (stereogram)] shows a vein (arrowheads) that appears to correspond to the enhancement through the facial canal on MRI is anteriorly connected to the dural sinus near the emissary vein of the foramen ovale (asterisk). It posterolaterally runs and then descends to flow into the external jugular vein (arrow).

aneurysm. The bone-subtracted CTA (Figs. 4A-4G) showed an unusual vein that traversed the right petrosal bone. The vein ran through the facial hiatus, and then ascended along the anterior wall of the petrosal bone and connected at the mid-part of the SPS. On the right carotid angiogram (Fig. 4H), the vein flowed to and fro along the anterior margin of the petrosal bone from the vicinity of the emissary vein of the foramen ovale.

\section{Discussion}

In our cases, unusual veins were situated in the petrosal bone. For the usual venous structures in the petrosal bone, the venous drainage of the inner ear is carried by the vein of the vestibular aqueduct into the sigmoid sinus near the endolymphatic sac, by the vein of the cochlear aqueduct into the extracranial IPS. ${ }^{2)}$ The internal auditory vein also participates in the venous drainage of the inner ear via the internal auditory meatus. It empties into the IPS, ${ }^{3)}$ the SPS or the transverse sinus, ${ }^{4)}$ or the great anterior cerebellar vein. $\left.{ }^{2}\right)$ The tympanic cavity can be drained by several veins accompanying the tympanic arteries and the branches of the facial or the glossopharyngeal nerve through the bony canals or apparatuses of the petrosal bone into the pterygoid plexus, the retromandibular vein, or the middle meningeal vein, such as the superior tympanic vein passing through the lessor petrosal canaliculus or the inferior tympanic vein passing through the tympanic canaliculus., ${ }^{2,45}$ The venous drainage of the facial nerve is chiefly carried rostrally by the superficial petrosal vein that accompanies the great superficial petrosal nerve into the lateral part of the cavernous sinus through the facial hiatus and caudally by the stylomastoid vein via the stylomastoid foramen into the retromandibular vein.,6) The internal carotid artery venous plexus is situated in the carotid canal enclosing the petrous internal carotid artery. It is rostrally connected to the inferior part of the cavernous sinus, caudally to the internal jugular vein forming one or more trunks. ${ }^{7)}$ The petrosquamosal sinus is a venous channel that is not constant but not so rare in the temporal bone. It posteriorly 


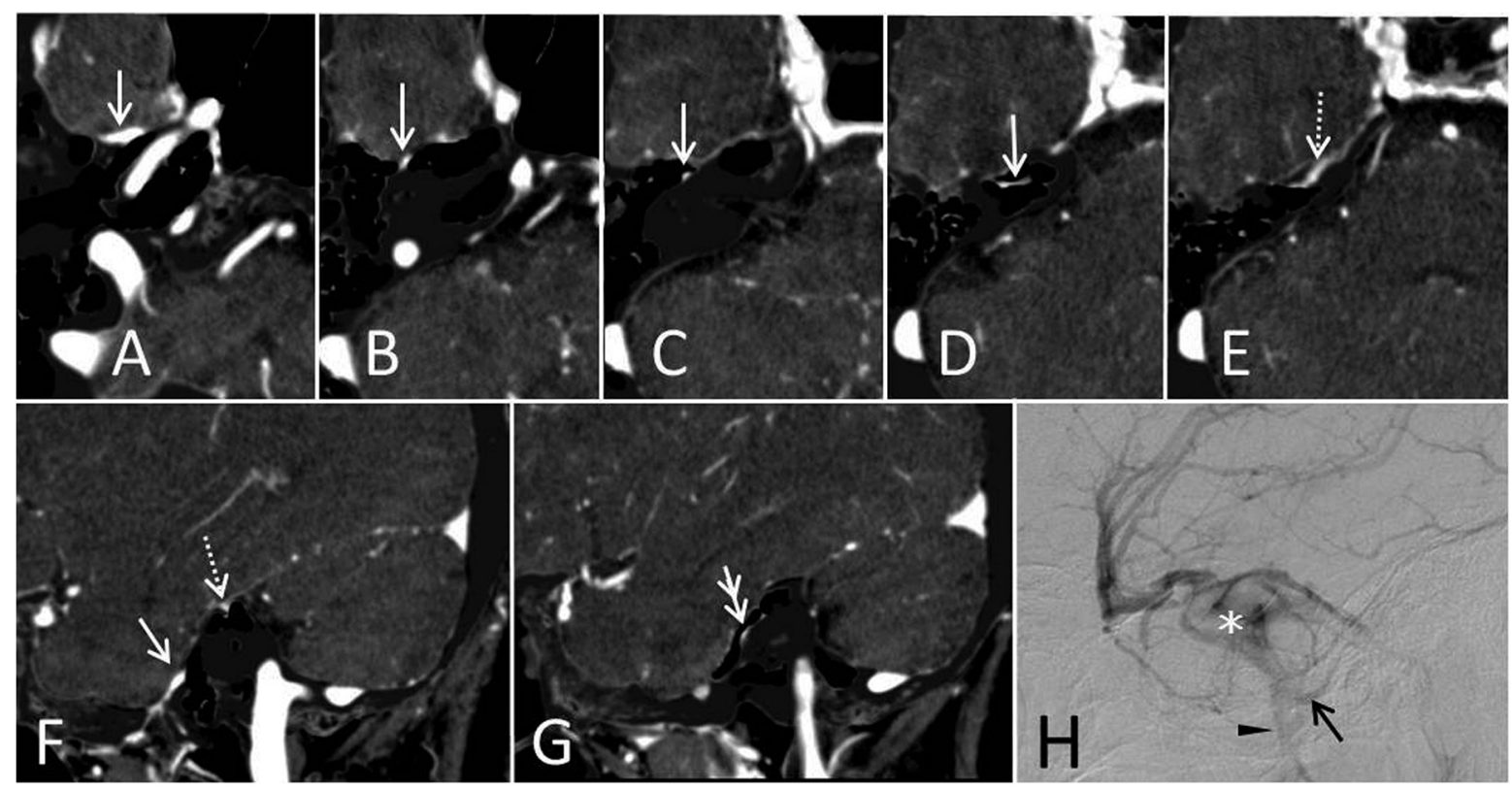

Fig. 4 Case 4. Axial bone-subtracted CTA around the right petrosal bone, from caudal (A) to cranial (E). (A, B) The vein (arrow) from the dural sinus near the foramen ovale enters the petrosal bone through the facial hiatus. (C) The vein (arrow) ascends along the anterior aspect of the petrosal bone. (D, E) The vein (arrow) exits the petrosal bone to connect with the superior petrosal sinus [SPS (dotted arrow)]. Sagittal reconstructed images of CTA, from medial (F) to lateral $(\mathbf{G})$ show that the vein enters into the petrosal bone through the facial hiatus (arrow) and ascends along the anterior wall (double arrow) of the petrosal bone to connect with the SPS (dotted arrow). (H) Venous phase of the right carotid angiogram (lateral view) shows that the vein (arrow) from the lateral part of the cavernous sinus (asterisk) near the emissary vein of the foramen ovale (arrowhead) flows to and fro along the anterior margin of the petrosal bone.

connects to the rostral portion of the transverse sinus, anteriorly runs along the junction of the squamous and petromastoid parts of the temporal bone, and empties into the retromandibular vein via the postglenoid foramen or the pterygoid plexus through the foramen ovale. ${ }^{8-10)}$ The petrosquamosal sinus is considered to be the remnant of the embryonic pro-otic sinus derived from the stem of the embryonic middle dural plexus (a tributary of the primary head sinus, cited later) that usually regresses during fetal or the early postnatal period. ${ }^{1)}$ The unusual veins in the petrosal bone in our cases could not be included in these typical venous variations in view of their size, running course, and drainage patterns (Fig. 5).

The temporal bone is a complex of bones with different embryological origins, and it is generally divided into three parts: squamous, tympanic, and petromastoid parts. ${ }^{4}$ The squamous part of the temporal bone is a membranous cranium for the brain. ${ }^{11-13)}$ The tympanic part develops from the embryonic tympanic ring through the intramembranous ossification and constitutes the anterior wall, floor, and part of the posterior wall of the external auditory meatus.4,11,14,15) The petromastoid part (petrosal bone) mainly develops from the embryonic otic capsule (future inner ear) through endochondral ossification. ${ }^{11-15)}$ The tympanic ring also contributes to the petrosal bone through the medial extension that lines its inferior surface to the carotid canal. ${ }^{4}$ Therefore the petrosal bone can be viewed as a complex of the cartilaginous neurocranium derived from the otic capsule and viscerocranium that covers the first pharyngeal pouch from which the tympanic cavity derived.

According to Padget, ${ }^{1)}$ early in the embryonic period ( $5 \mathrm{~mm}$ to $8 \mathrm{~mm}$ in length), a pair of primary head sinuses that drains all of the structures in the head region runs ventrolaterally to the primitive brain, dorsolaterally to the pharynx. It directly continues to the anterior cardinal vein (future internal jugular vein). The primitive brain originally drains through the primitive dural venous plexuses into the primary head sinus. The primitive dural plexuses were divided into three parts, the anterior, middle, and posterior dural plexuses, according to the corresponding area of the brain. The metencephalon (future cerebellum and pons) is chiefly drained by the middle dural plexus, which empties into the primary head sinus just caudal to the trigeminal nerve. The primary head sinus drains not only the brain but also the visceral structures that are ventrally 


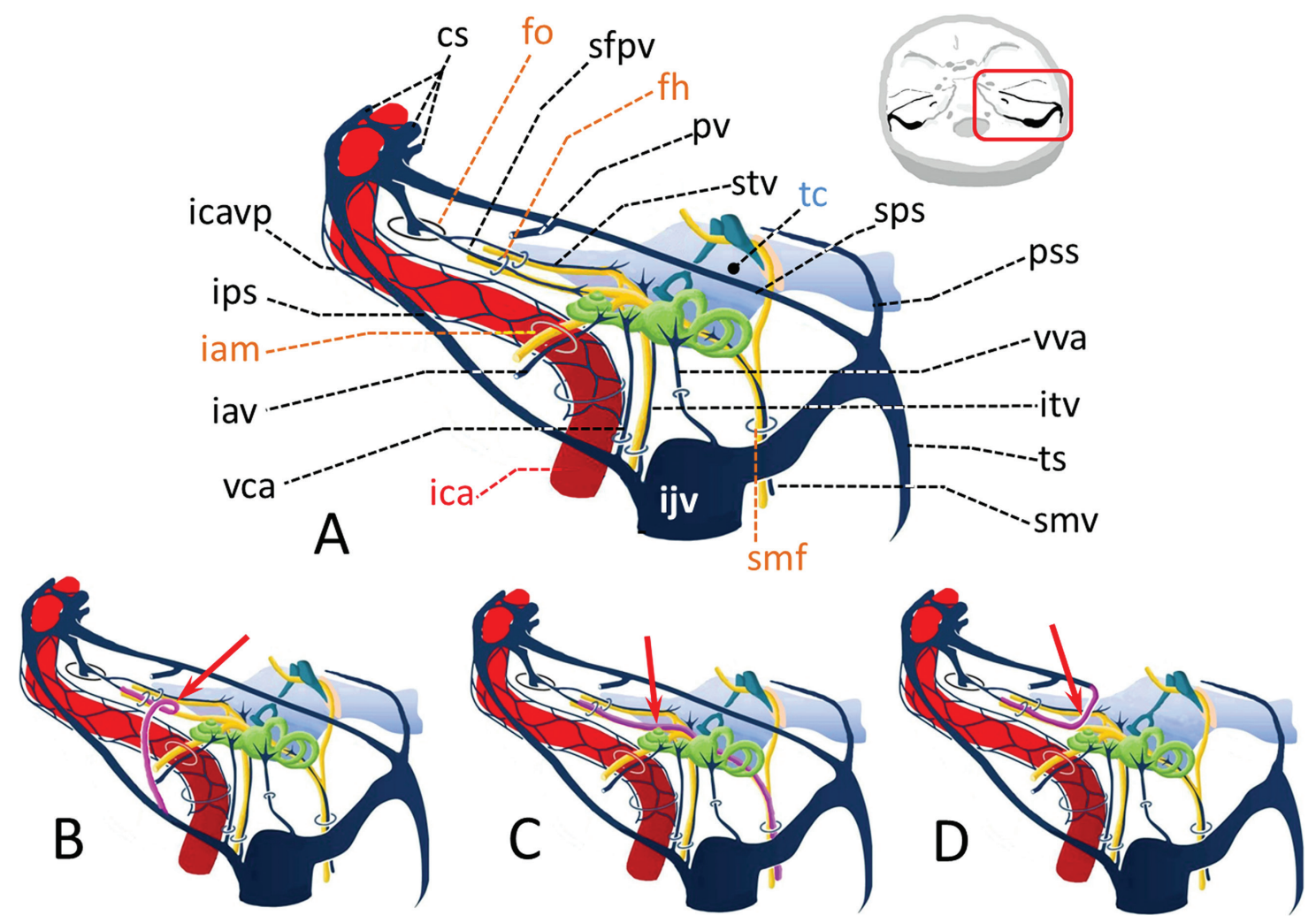

Fig. 5 (A) Schematic drawing of the normal venous structures in the petrosal bone (posterosuperior view). The inner ear is drained by the vein of the vestibular aqueduct (vva), the vein of cochlear aqueduct (vca), and the internal auditory vein (iav). The tympanic cavity is drained by the superior tympanic vein (stv), inferior tympanic vein (itv), and so on. The facial nerve is drained rostrally by the superficial petrosal vein (sfpv) through the facial hiatus (fh), caudally by the stylomastoid vein (smv) through the stylomastoid foramen (smf). The internal carotid artery venous plexus (icavp) is in the carotid canal enclosing the petrous internal carotid artery (ica). The petrosquamous sinus (pss) is situated in the junction of the squamous and petromastoid parts of the temporal bone. cs: cavernous sinus; fo: foramen ovale; iam: internal auditory meatus; ijv: internal jugular vein; ips: inferior petrosal sinus; pv: petrosal vein; sps: superior petrosal sinus; tc: tympanic cavity; ts: transverse sinus. (B-D) The running course of the veins (displayed in purple and indicated by red arrows) in the presented cases. (B) Cases 1 and 2. The vein that enters the petrosal bone through the facial hiatus crosses over the internal carotid artery and empties into the inferior petrosal sinus. (C) Case 3 . The vein runs entire length of the facial canal from the facial hiatus to the stylomastoid foramen. (D) Case 4. The vein enters the petrosal bone through the facial hiatus and ascends to reach the superior petrosal sinus.

situated to the brain, mainly through the dorsal pharyngeal vein (future emissary vein of the foramen ovale) that accompanies the third division of the trigeminal nerve. The primary head sinus is originally situated medially to the trigeminal and vagal nerves and laterally to the facial and glossopharyngeal nerves and otic capsule. By the time the embryo is $10 \mathrm{~mm}$ to $14 \mathrm{~mm}$ long, the primary head sinus laterally migrates to the vagal nerve. The original route of the primary head sinus when medial to the vagal nerve is a stem of the future IPS, which receives the ventral myelencephalic vein that drains the ventral part of the myelencephalon (future medulla oblongata). The caudal part of the primary head sinus situated laterally to the cranial nerves has also been called the lateral capital vein. When the embryo is $18 \mathrm{~mm}$ to $24 \mathrm{~mm}$ long, this caudal part of the primary head sinus that accompanies the facial nerve attenuates as being compressed between the enlarging otic capsule and facial nerve, paralleling the development of the new venous channel (sigmoid sinus) dorsal to the otic capsule. The remaining rostral part of the primary head sinus under the trigeminal nerve is the future cavernous sinus. The telencephalic vein (future superficial middle cerebral vein) that drains the ventrolateral part of the telencephalon into the anterior dural plexus migrates caudomedially accompanying the enlargement of the cerebral hemisphere and anastomoses to the cavernous sinus in the late fetal or postnatal period. These embryological osseous and venous developments are schematically shown in Figs. 6A-6C. When a portion of the primary head sinus which usually regresses during the embryonic stage remains in adults, it 

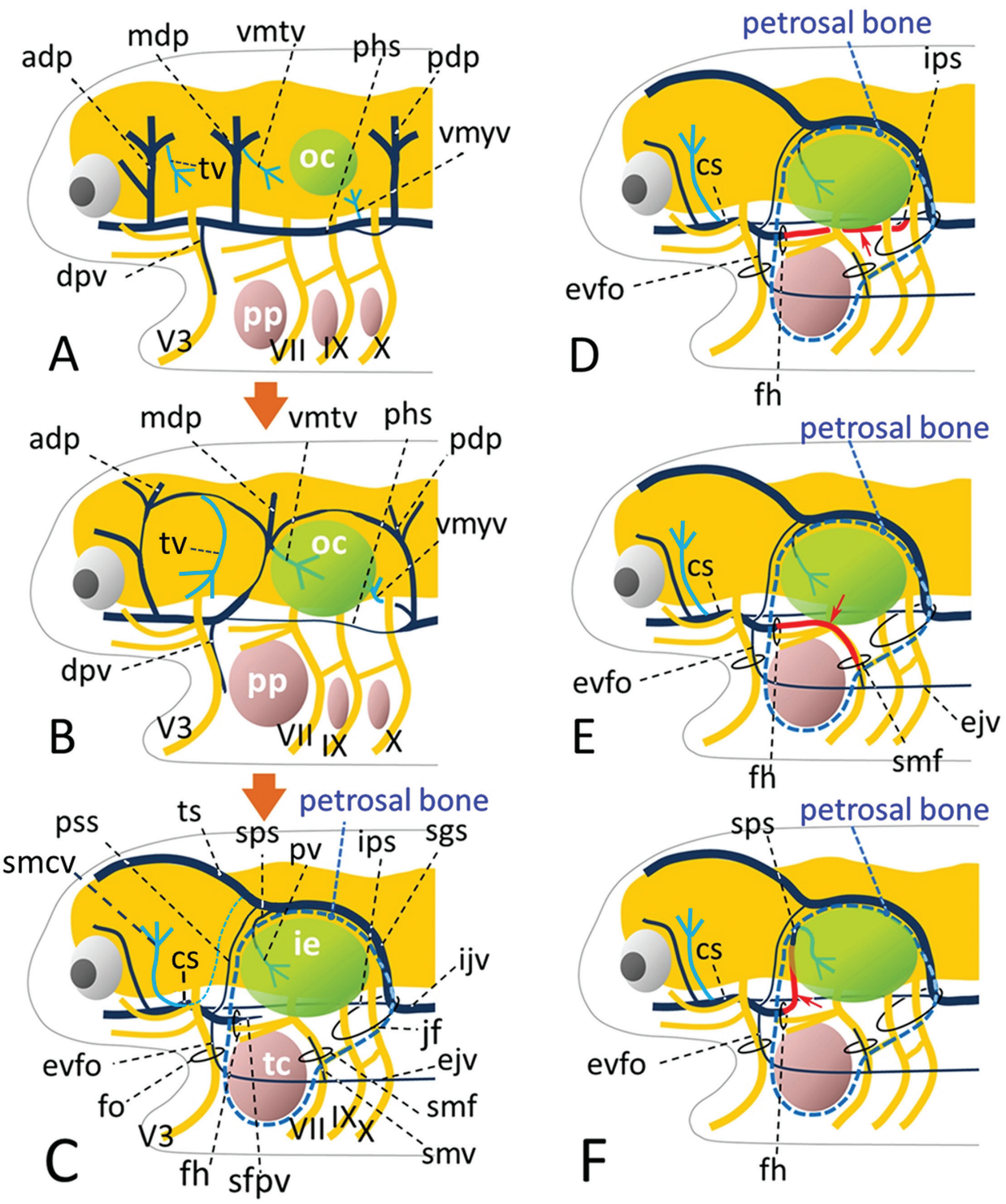

Fig. 6 (A-C) Conceptual schematic drawing of the venous development in the head region. (A) At the early embryonic stage $(5 \mathrm{~mm}$ to $8 \mathrm{~mm}$ in length), a pair of primary head sinuses situated ventrolaterally to the primitive brain carries the venous drainage of all structures in the head region. Each part of the primitive brain drains through the anterior, middle, and posterior dural plexuses into the primary head sinus. Subsequently, the primary head sinus situated medial to the vagal nerve that carries the venous drainage from the myelencephalon through the ventral myelencephalic vein laterally migrates to the vagal nerve. The ventral part of the metencephalon is drained by the ventral metencephalic vein into the middle dural plexus. (B) When the embryo is 10 to $14 \mathrm{~mm}$ in length, the primary head sinus completes the lateral migration to the vagal nerve. The part of the original channel medial to the vagal nerve continues to receive the ventral myelencepalic vein. The caudal end of the inferior petrosal sinus develops from this portion. When the embryo is $18 \mathrm{~mm}$ to $26 \mathrm{~mm}$ in length, as it accompanies the dorsal longitudinal anastomoses between the dural plexuses, the caudal part of the primary head sinus situated laterally to the facial, glossopharyngeal, and vagal nerves is attenuated as being compressed by the enlarging otic capsule (future inner ear), facial nerve and first pharyngeal pouch (future tympanic cavity). The telencephalic vein (future superficial middle cerebral vein) migrates caudomedially to anastomose to the cavernous sinus in the late fetal or postnatal period. (C) When the caudal part of the primary head sinus persists in an adult, it must be involved in the petrosal bone (blue dotted line). (D-F) Schematic drawings of the running course of the veins (red lines indicated by red arrows) in the presented cases. (D) Cases 1 and 2. The veins may be the remnants of the primary head sinus before the lateral migration to the vagal nerve. (E) Case 3 . The vein may be the remnant of the primary head sinus after the migration to the vagal nerve. (F) Case 4. The vein may be the remnant of the portion of the primary head sinus rostral to the entrance point of the ventral metencephalic vein that directly flows into the trunk of the primary head sinus and dural stem of the ventral metencephalic vein. adp: anterior dural plexus; cs: cavernous sinus; dpv: dorsal pharyngeal vein; ejv: external jugular vein; evfo: emissary vein of foramen ovale; fh: facial hiatus; fo: foramen ovale; ie: inner ear; ijv: internal jugular vein; ips: inferior petrosal sinus; jf: jugular foramen; mdp: middle dural plexus; oc: otic capsule; pdp: posterior dural plexus; phs: primary head sinus; pp: first pharyngeal pouch; pss: petrosquamosal sinus derived from the stem of the middle dural plexus; pv: petrosal vein; sfpv: superficial petrosal vein; sgs: sigmoid sinus; smcv: superficial middle cerebral vein; smf: stylomastoid foramen; smv: stylomastoid vein; sps: superior petrosal sinus derived from the ventral metencephalic vein; tc: tympanic cavity derived from first branchial pouch; ts: transverse sinus; tv: telencephalic vein; vmtv: ventral metencephalic vein; vmyv: ventral myelencephalic vein; V3: third division of the trigeminal nerve; VII: facial nerve; IX: glossopharyngeal nerve; $\mathrm{X}$ : vagal nerve 
is probably involved in the petrosal bone (to be precise, between the bones derived from the otic capsule and first pharyngeal pouch). It has been described ${ }^{1,2)}$ that the residual of the primary head sinus is just the lateral portion of the cavernous sinus connected to the emissary vein of the foramen ovale, superficial petrosal vein in the facial hiatus, and stylomastoid vein across the stylomastoid foramen.

The veins traversing the petrosal bone in all the four cases were anteriorly connected to the lateral part of the cavernous sinus near the foramen ovale through the facial hiatus. We then thought that these veins may be the remnants of the embryonic primary head sinus. In Cases 1 and 2 , the veins entered the petrosal bone through the facial hiatus and penetrated the petrous apex to empty into the caudal part of the IPS. The running course of the veins in these cases appeared similar to the course of the primary head sinus before lateral migration to the vagal nerve, except that it was medial to the facial and glossopharyngeal nerve. Butler demonstrated a case in which the dwindling primary head sinus ran medially to the facial nerve. $\left.{ }^{16}\right)$ This suggested that the primary head sinus occasionally migrates medially to the facial nerve during the developmental process. The veins in these cases may be the remnants of the primary head sinus before its migration lateral to the vagal nerve. In Case 3, the vein in the petrosal bone ran along the entire length of the facial canal from the facial hiatus to the stylomastoid foramen. This running course corresponds to the description about the residual of the primary head sinus by Padget ${ }^{1,2)}$ as mentioned above. In Case 4 , the vein entered the petrosal bone via the facial hiatus and dorsally ran to empty into the SPS. Padget ${ }^{1)}$ described that SPS is derived from the dural end of the embryonic ventral metencephalic vein that carries venous drainage from the embryonic metencephalon connecting the pial veins to the middle dural plexus as it traverses the differentiating subarachnoid layer (the subarachnoid part of the ventral metencephalic vein is the future petrosal vein). Therefore, the typical lateral end of SPS is usually at lateral portion of the transverse sinus which is derived from the middle dural plexus (Figs. 6A-6C). However, the ventral metencephalic vein may occasionally empty into the trunk of the primary head sinus. ${ }^{1)}$ When the ventral metencephalic vein empties into the primary head sinus trunk caudally to the entrance points of the middle dural plexus stem, the stem of the ventral metencephalic vein situated in the dural layer may be involved in the petrosal bone with the primary head sinus. Therefore we speculate that the vein in Case 4 is a remnant of the primary head sinus rostral to the entrance point of the ventral metencephalic vein and distal dural part of the ventral metencephalic vein. The considerations that these veins are the remnants of the primary head sinus are schematically shown in Figs. 6D-6F.

There have been some reports that have described venous anomalies in the petrosal bone concomitant with congenital dysplasia of the inner ear. ${ }^{17-20)}$ The development of the otic capsule may contribute to the regression of the primary head sinus, it then appears quite probable that congenital anomalies of the inner ear accompany the persistent primary head sinus and its tributaries. Nevertheless, most studies were based on just bone CT evaluations, and did not describe the course of the abnormal veins in detail. In those studies, the relevance of the venous anomaly to the embryonic primary head sinus remains unclear. We found three reports describing a dilated vein in the facial canal; ;,21,22) these cases were similar to the present Case 3. Nagar et al. ${ }^{22)}$ reported on a case with a dilated vein situated in the facial canal from the genu to the stylomastoid foramen and asserted that the vein was the remnant of the lateral capital vein. Herman et al. ${ }^{23)}$ reported aberrant vascular channels in the petrosal bone without any middle or inner ear abnormalities, as identified on CT and MRI, in four cases and speculated that these corresponded to a persistent lateral capital vein. Interestingly, in their four cases, the vascular channel did not appear to be related to the facial hiatus. In their cases, the aberrant vascular channels lay between the SPS sulcus and the sigmoid sinus sulcus or the posterior genu of the facial canal. These aberrant vascular channels that they reported may not be the remnant of the entire trunk of the primary head sinus in the region. Considering their continuity to SPS, these channels may be remnants of the dural end of the embryonic ventral metencephalic vein and a part (caudal to the entrance point of the ventral metencephalic vein) of the primary head sinus through a similar process as that was speculated for the vein in Case 4. In 2002, Kim et al. reported a case of an intraosseous arteriovenous fistula situated in the petrous apex and jugular fossa area. ${ }^{24)}$ It can be inferred that the arteriovenous fistula that they reported possibly developed in a similar primary head sinus remnant as we have presented in Cases 1 and 2. Recently, Mizutani et al. have reported an aberrant vein in the petrosal bone as a drainage route of a middle fossa dural arteriovenous fistula and considered that the vein may be the remnant of the primary 
head sinus based on its course in the petrosal bone from the facial hiatus to the jugular bulb. ${ }^{25)}$

In our cases, there were no congenital anomalies of the ear or abnormalities in the venous pathways around the petrosal bone which may have caused the persistence of the embryonic veins. The unusual veins in our cases were incidentally found during the evaluation of other intracranial disorders without related symptoms. Therefore, the exact cause and clinical importance of these veins are unclear. But, we think that the knowledge of the presence of these unusual venous variations in the petrosal bone that can be assumed to be the remnants of the primary head sinus may be important and useful to reach better understandings and therapeutic managements, when we encounter diseases that involve them such as arteriovenous fistulas. They might be the draining routes of the arteriovenous fistula, or the transvenous access routes for the embolization.

The limitation of this study is unclear prevalence of the veins as we presented, because we found the cases sporadically in several institutions with no consistent methodology. A further systematic investigation may be required.

\section{Conclusions}

We have reported on several unusual veins that traverse the petrosal bone. These veins may be the remnants of the embryonic primary head sinus and its tributaries, considering from their running course in the petrosal bone especially rostral communication with the lateral part of the cavernous sinus around the foramen ovale through the facial hiatus.

\section{Disclosure Statement}

The authors declare that they have no conflicts of interest.

\section{References}

1) Padget DH: The development of the cranial venous system in man, from the viewpoint of comparative anatomy. Contrib Embryol 1957; 36: 79-140.

2) Padget $\mathrm{DH}$ : The cranial venous system in man in reference to development, adult configuration, and relation to the arteries. Am J Anat 1956; 98: 307-355.

3) Mortazavi MM, Griessenauer CJ, Krishnamurthy S, et al: The inferior petrosal sinus: a comprehensive review with emphasis on clinical implications. Childs Nerv Syst 2014; 30: 831-834.

4) Williams PL, Bannister LH, Berry MM, et al: Gray's anatomy, 38th ed. Churchill Livingstone, Edinburgh, 1995, 1376-1396.

5) Moonis G, Mani K, O’Malley J, et al: A venous cause for facial canal enlargement: multidetector row CT findings and histopathologic correlation. AJNR Am J Neuroradiol 2011; 32: E83-E84.

6) Minatogawa T, Kumoi T, Hosomi H, et al: The blood supply of the facial nerve in the human temporal bone. Auris Nasus Larynx 1980; 7: 7-18.

7) Knott JF: On the cerebral sinuses and their variations. J Anat Physiol 1881; 16: 27-42.

8) San Millán Ruíz D, Gailloud P, Yilmaz H, et al: The petrosquamosal sinus in humans. J Anat 2006; 209: 711-720.

9) Zhao P, Wang Z, Xian J, et al: Persistent petrosquamosal sinus in adults: qualitative imaging evaluation on high-resolution CT venography. Acta Radiol 2014; 55: 225-230.

10) Marsot-Dupuch K, Gayet-Delacroix M, Elmaleh-Berges $\mathrm{M}$, et al: The petrosquamosal sinus: $\mathrm{CT}$ and MR findings of a rare emissary vein. AJNR Am J Neuroradiol 2001; 22: 1186-1193.

11) Abing W, Rauchfuss A: Fetal development of the tympanic part of the facial canal. Arch Otorhinolaryngol 1987; 243: 374-377.

12) Schoenwolf GC, Bleyl SB, Brauel PR, et al: Larsen's human embryology 4 th ed. Churchill Livingstone, London, 200, 545-550.

13) Romer AS, Parsons TS: The vertebrate body 6th ed. W.B. Saunders, Philadelphia 1986, 237-260.

14) Nemzek WR, Brodie HA, Chong BW, et al: Imaging findings of the developing temporal bone in fetal specimens. AJNR Am J Neuroradiol 1996; 17: 1467-1477.

15) Anson BJ: Developmental anatomy of the ear. In: otolaryngology, ed by Paparella MM, Schumrick DA. WB Saunders, Philadelphia, 1973, 3-74.

16) Butler H: The development of certain human dural venous sinuses. J Anat 1957; 91: 510-526.

17) Ozgen B, Oguz KK, Atas A, et al: Complete labyrinthine aplasia: clinical and radiologic findings with review of the literature. AJNR Am J Neuroradiol 2009; 30: 774-780.

18) Giesemann AM, Goetz GF, Neuburger J, et al: Persistent petrosquamosal sinus: high incidence in cases of complete aplasia of the semicircular canals. Radiology 2011; 259: 825-833.

19) Friedmann DR, Amoils M, Germiller JA, et al: Venous malformations of the temporal bone are a common feature in CHARGE syndrome. Laryngoscope 2012; 122: 895-900. 
20) Morimoto AK, Wiggins RH, Hudgins PA, et al: Absent semicircular canals in CHARGE syndrome: radiologic spectrum of findings. AJNR Am J Neuroradiol 2006; 27: $1663-1671$.

21) Kim M, Lim DW, Lee HY, et al: Venous engorgement as a cause of facial canal enlargement. Int Adv Otol 2014; 10: 184-186.

22) Nager GT, Proctor B: Anatomic variations and anomalies involving the facial canal. Otolaryngol Clin North Am 1991; 24: 531-553.
23) Hermans R, van Rensburg LJ: An aberrant vascular channel in the petrous bone: persistent lateral capital vein? Eur Radiol 2009; 19: 2958-2964.

24) Kim MS, Oh CW, Han DH, et al: Intraosseous dural arteriovenous fistula of the skull base associated with hearing loss. Case report. J Neurosurg 2002; 96: 952-955.

25) Mizutani K, Akiyama T, Yoshida K: The remnant of primary head sinus found in the case of dural arteriovenous fistula: A case report. INR interventional neuroradiology 2016; DOI: 10.1177/1591019916641903. 